\title{
Rare decays of $B, D$ and $K$ mesons
}

\author{
Niels Tuning ${ }^{* \dagger}$ \\ Nikhef \& CERN \\ E-mail: tuning@nikhef.nl
}

Rare decays of beauty, charm and strange hadrons are particularly sensitive to contributions from beyond the Standard Model. Results on branching fractions, decay rates, asymmetries and lepton flavour non-universality of decays with leptons in the final state are presented. These results are obtained from numerous experimental facilities.

ICHEP 2018, XXXIX International Conference on High Energy Physics

4-11 July 2018

Seoul, South Corea

* Speaker.

${ }^{\dagger}$ On behalf of the LHCb collaboration, with material from ATLAS, BaBar, Belle, BESIII, CMS, KOTO, NA62 


\section{Introduction}

Flavour physics in general and rare decays in particular are sensitive probes to scrutinize the Standard Model (SM). As the SM amplitudes of rare decays are suppressed, any small new physics effects (NP) can compete in magnitude. Historically such precision measurements have led to remarkable discoveries, for example the measurements of the

- (lack of) $K^{0} \rightarrow \mu^{+} \mu^{-}$decays [1], which led to the introduction of charm;

- discovery of CP violation [2], which led to the prediction of the $3^{\text {rd }}$ family like bottom [3];

- measurement of $B^{0}$ mixing [4], which led to the prediction of the top mass, $m_{t}>50 \mathrm{GeV}$.

In these proceedings the latest measurements on rare $B, D$ and $K$ decays are presented, which all fall in the class of flavour changing neutral current (FCNC) electroweak penguin (EWP) processes, as illustrated in Fig. 1. FCNC are not allowed at tree level in the SM and are thus loopsuppressed whereas new heavy particles can contribute significantly in these loop processes. Depending on the coupling strength, mass scales between a few TeV (for Standard Model-like couplings known as minimal flavour violation (MFV)) and few tens of $\mathrm{TeV}$ (for couplings of order unity) can be probed, with the current experimental accuracies.
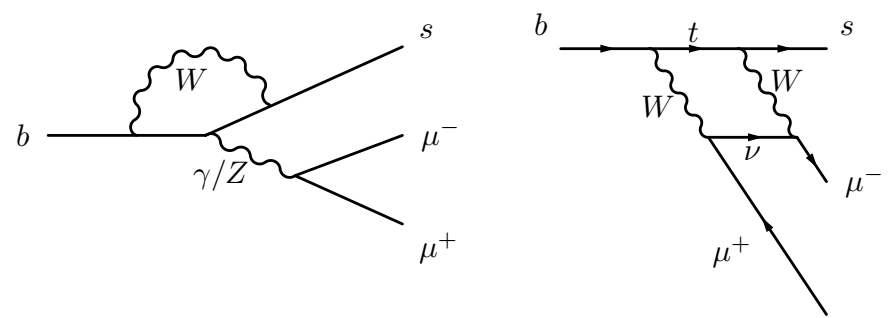

Figure 1: Flavour Changing Neutral Current processes in the Standard Model occur at loop level. The $s \rightarrow d \ell \ell$ and $c \rightarrow u \ell \ell$ processes are similar to the $b \rightarrow s \mu^{+} \mu^{-}$diagrams shown here.

These FCNC processes can be realized with a large variety of hadrons, leading to the study of numerous decays. The decays that are discussed in these proceedings are tabulated in Tab. 1.

\begin{tabular}{l||l|l|l}
\hline & Fully Leptonic & \multicolumn{2}{|c}{ Rare semileptonic } \\
& & Mesonic & Baryonic \\
\hline \hline Strange & $K_{S}^{0} \rightarrow \mu^{+} \mu^{-}$ & $K^{+} \rightarrow \pi^{+} v \bar{v}$ & $\Sigma^{+} \rightarrow p \mu^{+} \mu^{-}$ \\
& & $K^{0} \rightarrow \pi^{0} v \bar{v}$ & $\psi(2 S) \rightarrow \Lambda_{c}^{+} p e^{+} e^{-}$ \\
\hline Charm & & $D \rightarrow h h^{\prime} \mu^{+} \mu^{-}$ & $\Lambda_{c}^{+} \rightarrow p \mu^{+} \mu^{-}$ \\
& & $\psi \rightarrow D^{0} e^{+} e^{-}$ & \\
\hline Bottom & $B_{(s)}^{0} \rightarrow \mu^{+} \mu^{-}$ & $B \rightarrow K^{(*)} \mu^{+} \mu^{-}$ & $\Lambda_{b}^{0} \rightarrow \Lambda \mu^{+} \mu^{-}$ \\
& $B_{(s)}^{0} \rightarrow \tau^{+} \tau^{-}$ & $B \rightarrow K^{(*)} e^{+} e^{-}$ & \\
& $B_{(s)}^{0} \rightarrow \mu^{+} e^{-}$ & $B_{s}^{0} \rightarrow\left(K^{*} / \phi\right) \mu^{+} \mu^{-}$ & \\
\hline
\end{tabular}

Table 1: Results on these decays are presented in this paper. 


\section{Strange}

\subsection{Leptonic: $K_{S}^{0} \rightarrow \mu^{+} \mu^{-}$}

Research on decays of hadrons containing strange quarks dates back to the very discovery of long-living particles in 1947 [5]. The $K^{0} \rightarrow \ell^{+} \ell^{-}$decay amplitude receives S-wave $(A)$ and P-wave $(B)$ components, $\mathscr{A}\left(K^{0} \rightarrow \ell^{+} \ell^{-}\right)=\bar{u}_{l}\left(i B+A \gamma_{5}\right) v_{l}$ [6], resulting in different expectations for $K_{L}^{0}$ and $K_{S}^{0}$ decays. Long-distance contributions to $K_{S}^{0} \rightarrow \mu^{+} \mu^{-}$decays are small compared to the $K_{L}^{0}$ case, which makes the study of $K_{S}^{0}$ decays sensitive to new physics. Recently, the LHCb experiment entered this area with a new upper limit on the branching fraction,

$$
\mathscr{B}\left(K_{S}^{0} \rightarrow \mu^{+} \mu^{-}\right)<0.8 \times 10^{-9} \text { at } 90 \% \mathrm{CL} \text { [7] }
$$

This improves on an earlier LHCb measurement and constitutes the worlds best limit.

\subsection{Rare semileptonic: $K \rightarrow \pi v \bar{v}$ and $\Sigma^{+} \rightarrow p \mu^{+} \mu^{-}$decays}

The NA62 experiment at the SPS observed a single $K^{+} \rightarrow \pi^{+} v \bar{v}$ candidate, whereas 0.27 signal events and 0.15 background events were expected. The KOTO experiment at J-PARC did not observe any neutral kaon decays, leading to new limits on the branching fraction,

$$
\begin{aligned}
\mathscr{B}\left(K^{+} \rightarrow \pi^{+} v \bar{v}\right) & <14 \times 10^{-10} \text { at } 95 \% \mathrm{CL}[8], \\
\mathscr{B}\left(K_{L}^{0} \rightarrow \pi^{0} v \bar{v}\right) & <30 \times 10^{-10} \text { at } 90 \% \mathrm{CL}[9],
\end{aligned}
$$

while the SM expectations for the branching fractions are $0.84 \times 10^{-10}$ and $0.3 \times 10^{-10}$ for the $K^{+}$ and $K_{L}^{0}$ decay, respectively [10]. By 2019 the NA62 collaboration might have analyzed their total available dataset, with approximately 20 expected $K^{+}$decays, according to Standard Model predictions, whereas the KOTO collaboration expects to improve the upper limit on the $K_{L}^{0}$ branching fraction to $12 \times 10^{-10}$.

The LHCb collaboration further contributed to the study of rare decays of hadrons containing strange quarks by studying the baryonic decay $\Sigma^{+} \rightarrow p \mu^{+} \mu^{-}$. A resonant structure in the dimuon invariant mass around $214 \mathrm{MeV}$, close to the dimuon threshold, was reported by the HyperCP experiment with the observation of three events [11]. No evidence for such an exotic scalar particle $X$ is found by $\mathrm{LHCb}$,

$$
\begin{aligned}
\mathscr{B}\left(\Sigma^{+} \rightarrow p \mu^{+} \mu^{-}\right) & =\left(2.2_{-1.3}^{+1.8}\right) \times 10^{-8} \\
\mathscr{B}\left(\Sigma^{+} \rightarrow p X\left(\rightarrow \mu^{+} \mu^{-}\right)\right) & <1.4 \times 10^{-8} \text { at } 90 \% \text { CL [12]. }
\end{aligned}
$$




\section{Charm}

\subsection{Rare semileptonic: $D^{0}, \psi$ and $\Lambda_{c}$ decays}

Rare charm decays are of interest as down-type quarks constitute to the loop diagrams, in contrast to $B$ or $K$ decays, and new particles might in principle couple differently to the up-quark sector. The rarest charm decay ever observed is reported,

$$
\begin{aligned}
\mathscr{B}\left(D^{0} \rightarrow \pi^{+} \pi^{-} \mu^{+} \mu^{-}\right) & =(9.6 \pm 1.2) \times 10^{-7}, \\
\left.\mathscr{B} D^{0} \rightarrow K^{+} K^{-} \mu^{+} \mu^{-}\right) & =(1.54 \pm 0.33) \times 10^{-7}[13] .
\end{aligned}
$$

The addition of the data set recorded in 2015 and 2016 by LHCb allowed for an angular analysis, showing that the $\mathrm{CP}$ - and forward-backward asymmetries are consistent with zero [14].

No significant signal is observed of the baryonic decay,

$$
\mathscr{B}\left(\Lambda_{c}^{+} \rightarrow p \mu^{+} \mu^{-}\right)<7.7 \times 10^{-8} \text { at } 90 \% \mathrm{CL}[15],
$$

where the regions of the dimuon mass around the $\rho^{0} / \omega$ and $\phi$ resonances are used as normalisation.

The BESIII collaboration also studied FCNC decays of $D$-mesons, but with an electron pair in the final state, and upper limits are set in the following ranges,

$$
\begin{aligned}
\mathscr{B}\left(D^{+} \rightarrow h^{ \pm} e^{+} e^{\mp}\right) & <[3-12] \times 10^{-5} \text { at } 90 \% \mathrm{CL}, \\
\mathscr{B}\left(D^{+} \rightarrow h^{+} h^{0} e^{+} e^{-}\right) & <[1.1-2.6] \times 10^{-5} \text { at } 90 \% \mathrm{CL}, \\
\mathscr{B}\left(D^{0} \rightarrow h^{+} h^{-} e^{+} e^{-}\right) & <[0.7-4.1] \times 10^{-5} \text { at } 90 \% \mathrm{CL}, \\
\mathscr{B}\left(D^{0} \rightarrow h^{0} e^{+} e^{-}\right) & <[0.3-1.2] \times 10^{-5} \text { at } 90 \% \mathrm{CL}[16],
\end{aligned}
$$

where $h=\pi, K$. Similarly, rare charm decays can be studied with charmonium decays, i.e. with a charm quark also as spectator quark, and the following limits on branching fractions of $\psi$ decays to $e^{+} e^{-}$pairs are set,

$$
\begin{aligned}
\mathscr{B}\left(J / \psi \rightarrow D^{0} e^{+} e^{-}\right) & <8.5 \times 10^{-8} \text { at } 90 \% \mathrm{CL}, \\
\mathscr{B}\left(\psi(2 S) \rightarrow D^{0} e^{+} e^{-}\right) & <14 \times 10^{-8} \text { at } 90 \% \mathrm{CL}[17], \\
\mathscr{B}\left(\psi(2 S) \rightarrow \Lambda_{c}^{-} p e^{+} e^{-}\right) & <170 \times 10^{-8} \text { at } 90 \% \mathrm{CL}[18] .
\end{aligned}
$$




\section{Beauty}

4.1 Leptonic: $B_{(s)}^{0} \rightarrow \mu^{+} \mu^{-}, B_{(s)}^{0} \rightarrow \tau^{+} \tau^{-}$and $B_{(s)}^{0} \rightarrow e^{+} \mu^{-}$

The quest for the $B_{s}^{0} \rightarrow \mu^{+} \mu^{-}$lasted for many decades, culminating in its observation in 2012 [19]. It arguably forms the most important result of the LHC after the Higgs discovery, as the SM prediction is very precise, and any deviation can be a hint of the existence of e.g. new scalar particles in the MSSM framework [20]. The latest results include data from the LHCb collaboration recorded in 2015 and 2016,

$$
\begin{aligned}
& \mathscr{B}\left(B_{s}^{0} \rightarrow \mu^{+} \mu^{-}\right)=\left(3.0 \pm 0.6_{-0.2}^{+0.3}\right) \times 10^{-9}, \\
& \mathscr{B}\left(B^{0} \rightarrow \mu^{+} \mu^{-}\right)<0.34 \times 10^{-9} \text { at } 95 \% \mathrm{CL},[21]
\end{aligned}
$$

where the largest contribution to the systematic uncertainty originates from the knowledge on the production rate of $B_{s}^{0}$ mesons [22]. New scalar couplings could potentially leave the branching fraction unchanged, while affecting the $\mathrm{CP}$ structure. A novel CP-even component would be visible as a reduction in the measured lifetime [20], while the SM predicts a lifetime corresponding to the lifetime of the heavy (CP-odd) $B_{s}$ mass eigenstate, $\tau_{B_{s}^{0} \rightarrow \mu^{+} \mu^{-}}=\tau_{H}=1.658 \mathrm{ps}$. A first measurement of the effective lifetime is reported,

$$
\tau\left(B_{s}^{0} \rightarrow \mu^{+} \mu^{-}\right)=2.04 \pm 0.44 \pm 0.05 \text { ps }[21] .
$$

Decays to $\tau$ lepton pairs are particularly interesting, as the helicity suppression is largely lifted in the SM, and because new particles might couple differently to the third generation. Experimentally however, the measurement is challenging due to the missing neutrinos, and due to the penalty of the subsequent $\tau$ branching fraction,

$$
\begin{aligned}
& \mathscr{B}\left(B_{s}^{0} \rightarrow \tau^{+} \tau^{-}\right)<5.2 \times 10^{-3} \text { at } 90 \% \mathrm{CL}, \\
& \mathscr{B}\left(B^{0} \rightarrow \tau^{+} \tau^{-}\right)<1.6 \times 10^{-3} \text { at } 95 \% \mathrm{CL} \text { [23]. }
\end{aligned}
$$

while the SM prediction is $(7.7 \pm 0.5) \times 10^{-7}$ and $(2.2 \pm 0.2) \times 10^{-7}$ for $B_{s}^{0}$ and $B^{0}$, respectively [24]. Models with vector leptoquarks that couple to the third generation predict branching fractions as large as $10^{-4}[25]$.

Recently renewed interest has arisen in the search for lepton flavour violating (LFV) decays, as it has been argued that lepton flavour non-universality (LFNU) implies LFV [26]. The following limits have been set,

$$
\begin{aligned}
& \mathscr{B}\left(B_{s}^{0} \rightarrow e^{+} \mu^{-}\right)<6.0 \times 10^{-9} \text { at } 90 \% \mathrm{CL}, \\
& \mathscr{B}\left(B^{0} \rightarrow e^{+} \mu^{-}\right)<1.0 \times 10^{-9} \text { at } 90 \% \mathrm{CL} \text { [27]. }
\end{aligned}
$$




\subsection{Rare semileptonic: $B \rightarrow K \mu^{+} \mu^{-}, B_{s}^{0} \rightarrow \phi \mu^{+} \mu^{-}$and $\Lambda_{b}^{0} \rightarrow \Lambda^{0} \mu^{+} \mu^{-}$decays}

The $b \rightarrow s \mu^{+} \mu^{-}$process can be studied with a variety of hadronic decay modes. The decay rates of the decays $B^{+} \rightarrow K^{+} \mu^{+} \mu^{-}, B^{0} \rightarrow K^{* 0} \mu^{+} \mu^{-}, B_{s}^{0} \rightarrow \phi \mu^{+} \mu^{-}$and $\Lambda_{b}^{0} \rightarrow \Lambda^{0} \mu^{+} \mu^{-}$are measured consistently below expectations.

A particularly rich experimental system is provided by $B^{0} \rightarrow K^{* 0} \mu^{+} \mu^{-}$decays. In addition to the decay rate (as a function of the dimuon invariant mass squared $q^{2}$ ), numerous angular observables, like the forward-backward asymmetry and other angular asymmetries, can be compared to SM expectations. Many theoretical uncertainties cancel in the determination of angular asymmetries, resulting in accurate probes of new physics effects. Moreover, angular observables can be used to determine the type of the coupling of any new physics contribution, being sensitive to the Lorentz structure.

Numerous experiments have performed angular analyses, notably from the BaBar [29], $\mathrm{LHCb}$ [30] ATLAS [31] CMS [32,33] and Belle [37] experiments, as shown in the left of Fig. 2. A particularly interesting angular observable is the $S_{5}$ asymmetry, which sums the forward/upward with backward/downward going kaons, and quantifies the asymmetry with the complementary event sample. The $P_{5}^{\prime}$ observable (defined in Ref. [34] and references therein) is less dependent on form factors and is obtained by dividing $S_{5}$ by $\sqrt{F_{T} F_{L}}$. The angular observables - most notably $P_{5}^{\prime}$, combined with the decay rate measurements, shows a tension with the SM expectations. Global fits to about 200 measurements of the $b \rightarrow s \mu^{+} \mu^{-}$process from all aforementioned experiments show a significant improvement in the fit quality of about $\Delta \chi^{2} \sim 25$ (e.g. in Ref.[38]). This is obtained when beyond-the-SM vector $(V)$ or vector-axial $V-A$ couplings are allowed for, quantified through the effective coupling strengths $C_{9}^{N P}$ and $C_{9}^{N P}-C_{10}^{N P}$, respectively. These so-called Wilson coefficients quantify the left-handed couplings to the quarks, whereas the right-handed couplings to the quarks are usually indicated by the $C_{i}^{\prime}$ coefficients. It is an appealing feature that it is possible to consistently explain all deviating measurements by a single, simple, adjustement of either the vector, or $V-A$ coupling. This eases the model building to provide possible explanations of the observations, like models containing $Z^{\prime}$ bosons or leptoquarks (see for example Ref. [25] and references therein).

Recently, also the FCNC $b \rightarrow d \mu^{+} \mu^{-}$electroweak penguin process was probed in $B_{s}^{0}$ decays,

$$
\mathscr{B}\left(B_{s}^{0} \rightarrow K^{* 0} \mu^{+} \mu^{-}\right)=(2.9 \pm 1.1) \times 10^{-8}[28],
$$

which could provide a very interesting comparison to the angular observables in the $B^{0} \rightarrow K^{* 0} \mu^{+} \mu^{-}$ case, once a sufficiently large event sample will be available.

\subsection{Lepton Flavour Non-Universality}

The excitement of the collection of small deviations in the $b \rightarrow s \mu^{+} \mu^{-}$decay rates and angular observables is augmented by theoretically clean tests of lepton flavour universality in rare decays, quantified through the ratio of $\mu^{+} \mu^{-}$over $e^{+} e^{-}$decay rates,

$$
\begin{aligned}
R_{K} & =0.745_{-0.074}^{+0.09} \pm 0.036[35], \\
R_{K *} & =0.66_{-0.07}^{+0.11} \pm 0.03[36] \\
R_{K *} & =0.69_{-0.07}^{+0.11} \pm 0.05[36]
\end{aligned}
$$$$
\text { for } 0.045<q^{2}<1.1 \mathrm{GeV}^{2} \text {, }
$$$$
\text { for } 1.1<q^{2}<6.0 \mathrm{GeV}^{2} \text {. }
$$ 

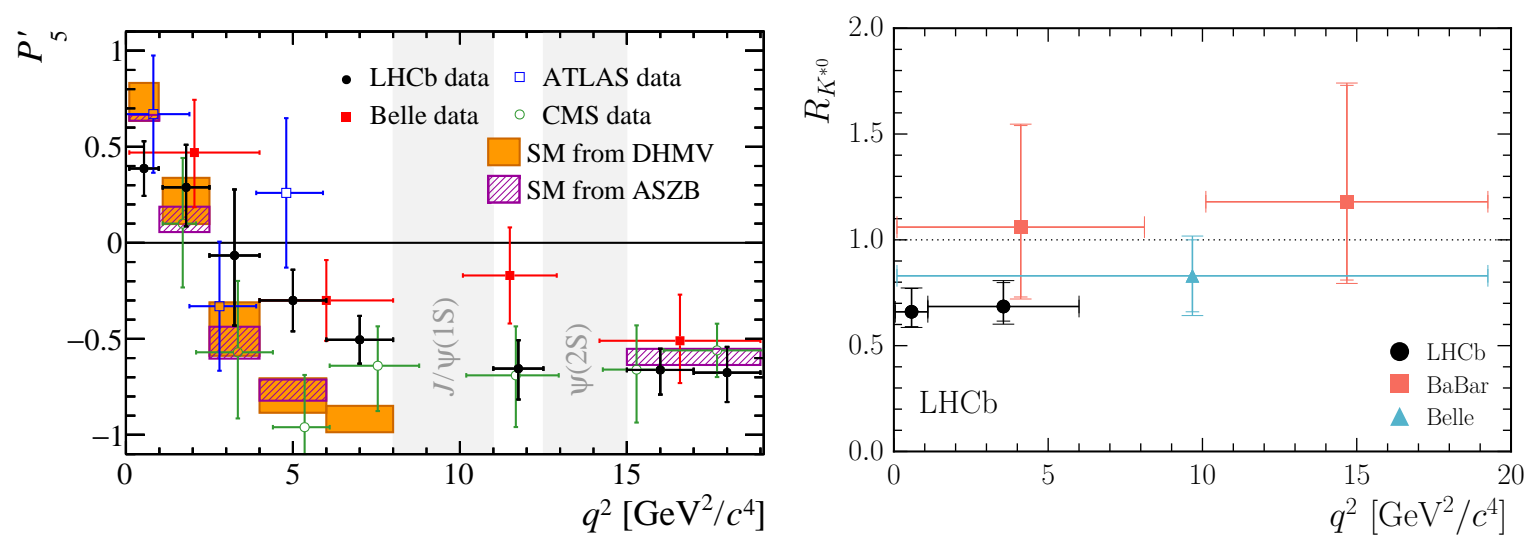

Figure 2: Compilation of results on $b \rightarrow s \ell \ell$ flavour-changing neutral-current decays: $P_{5}^{\prime}$ (left) and $R_{K}^{*}$ (right) as a function of the invariant mass squared $q^{2}$ of the lepton pair.

The SM predicts ratios close to unity, with very small uncertainty, as most theoretical uncertainties originating from form factors or charm-loop effects, cancel in the ratio. The measurements on $R_{K *}$ are shown in the right of Fig. 2. However, it is difficult to explain the deviation (albeit only 2.2 standard deviations) from unity of the ratio $R_{K *}$ in the low- $q^{2}$ bin, even when invoking new physics. As the low- $q^{2}$ bin is dominated by the photon pole (quantified through the Wilson coefficient $C_{7}$ ), and as it is known that the photon couples equally to charged particles, new physics effects are not expected to have a large impact in this $q^{2}$ region.

Experimentally many possible systematics cancel, because $R_{K(*)}$ is determined as double ratio, normalized to the $B \rightarrow K^{(*)} J / \psi(\ell \ell)$ decay rates. The single ratio $R_{J / \psi}=\mathscr{B}\left(B^{0} \rightarrow K^{* 0} J / \psi(\rightarrow\right.$ $\left.\left.\mu^{+} \mu^{-}\right)\right) / \mathscr{B}\left(B^{0} \rightarrow K^{* 0} J / \psi\left(\rightarrow e^{+} e^{-}\right)\right)$is less precise, but forms an extremely stringent test, and is studied as a function of numerous kinematic quantities. The average ratio is determined to be $R_{J / \psi}=1.043 \pm 0.006$ (stat) \pm 0.045 (sys). The double ratio using the $K^{*} \psi(2 S)$ final state (in place of $K^{*} \ell \ell$ ) is measured to be consistent with unity within $1 \sigma$ of the statistical uncertainty of $2 \%$.

An angular analysis of $B^{0} \rightarrow K^{*} e^{+} e^{-}$would give important complementary information, and could possibly reconcile the intriguing measurements of the angular distributions of $B^{0} \rightarrow K^{*} \mu^{+} \mu^{-}$ decays and the low decay rates of $B \rightarrow K \mu^{+} \mu^{-}$decays compared to $B \rightarrow K e^{+} e^{-}$decays.

The Belle collaboration has indeed compared the anomalous $P_{5}^{\prime}$ measurement with muons in the final state, with the measurement using electrons. The current precision does not allow to draw firm conclusions yet, but it is interesting to note that the $P_{5}^{\prime}$ measurement with electrons agrees better with the SM predictions [37].

Combined with the deviating measurements of the ratio of semileptonic $B \rightarrow D^{(*)} \tau \nu$ over $B \rightarrow$ $D^{(*)} \ell v, \ell=\mu, e$ decays, it is tempting to speculate on new physics scenarios where new particles couple mainly to the $\tau$ leptons (even competing with tree level SM semileptonic decays), and to a lesser extent to muons (thus competing with SM loop processes), and negligibly to electrons (see e.g. Refs. $[25,26]$ and references therein). 


\section{Conclusions and outlook}

A world-wide effort is ongoing in the area of flavour-changing neutral-currents, most notably regarding $b \rightarrow$ sll electroweak penguin processes. Intriguing tensions with the Standard Model have been observed, while showing a consistent picture among the experimental data from numerous different experiments.

Both the historical measurements (e.g. on $K^{0} \rightarrow \mu^{+} \mu^{-}$or $B^{0}$-mixing), and the current excitement on rare beauty decays, clearly shows the power of precision flavour physics, being sensitive to phenomena at multi-TeV energy scales. In addition, the novel angular analysis of charm decays promises a new window on possible new physics effects in the up-quark sector.

The sensitivities of all measurements presented here are dominated by the statistical uncertainties, which justifies increased efforts in future flavour experiments, like the upcoming LHCb Upgrade I and Belle II experiments, the optimization of the triggers strategies in ATLAS and CMS, and the plans for a future LHCb Upgrade II [39]. Table 2 illustrates the potential of the future dedicated flavour physics facilities, in terms of event yields of $B^{0} \rightarrow K^{* 0} \mu^{+} \mu^{-}$and $B^{0} \rightarrow K^{* 0} e^{+} e^{-}$ decays.

\begin{tabular}{|c|c|c|c|c|c|c|c|}
\hline \multirow{3}{*}{$\mathrm{LHCb}$} & 2010 & 2014 & 2019 & 2024 & 2026 & 2030 & \multirow{2}{*}{\begin{tabular}{|l}
2040 \\
Upgrade II
\end{tabular}} \\
\hline & & & & \multicolumn{3}{|c|}{ Upgrade I } & \\
\hline & & $\begin{array}{l}\text { Run-1 } \\
3 \mathrm{fb}^{-1} \\
111(2500)\end{array}$ & $\begin{array}{l}\text { Run-2 } \\
9 \mathrm{fb}^{-1} \\
0.5(10) \mathrm{k}\end{array}$ & $\begin{array}{l}\text { Run-3 } \\
23 \mathrm{fb}^{-1} \\
1.4(30) \mathrm{k}\end{array}$ & & $\begin{array}{l}\text { Run-4 } \\
50 \mathrm{fb}^{-1} \\
3.3(70) \mathrm{k}\end{array}$ & $\begin{array}{l}300 \mathrm{fb}^{-1} \\
20(400) \mathrm{k}\end{array}$ \\
\hline Belle & $\begin{array}{l}\text { Belle I } \\
0.7 \mathrm{ab}^{-1} \\
127(185)\end{array}$ & & & & $\begin{array}{l}\text { Belle II } \\
50 \mathrm{ab}^{-1} \\
10(13) \mathrm{k}\end{array}$ & & \\
\hline
\end{tabular}

Table 2: Comparison of estimated event yields for the decays $B^{0} \rightarrow K^{* 0} e^{+} e^{-}\left(\mu^{+} \mu^{-}\right)$. The LHCb estimates are taken from Ref. [39], whereas the Belle yields are taken from Ref. [37], and scaled to $50 \mathrm{ab}^{-1}$ using the relative luminosity. 


\section{References}

[1] S. L. Glashow, J. Iliopoulos and L. Maiani, "Weak Interactions with Lepton-Hadron Symmetry," Phys. Rev. D 2 (1970) 1285.

[2] J. H. Christenson, J. W. Cronin, V. L. Fitch and R. Turlay, "Evidence for the $2 \pi$ Decay of the $K_{2}^{0}$ Meson,” Phys. Rev. Lett. 13 (1964) 138.

[3] M. Kobayashi and T. Maskawa, "CP Violation in the Renormalizable Theory of Weak Interaction," Prog. Theor. Phys. 49 (1973) 652.

[4] H. Albrecht et al. [ARGUS coll.], “Observation of B0 - anti-B0 Mixing,” Phys. Lett. B 192 (1987) 245.

[5] G. D. Rochester and C. C. Butler, "Evidence for the Existence of New Unstable Elementary Particles," Nature 160 (1947) 855.

[6] G. Isidori and R. Unterdorfer, "On the short distance constraints from $K(L, S) \rightarrow \mu^{+} \mu^{-}$," JHEP 0401 (2004) 009 [hep-ph/0311084].

[7] R. Aaij et al. [LHCb coll.], "Improved limit on the branching fraction of the rare decay $K_{\mathrm{S}}^{0} \rightarrow \mu^{+} \mu^{-}$," Eur. Phys. J. C 77 (2017) no.10, 678 [arXiv:1706.00758 [hep-ex]].

[8] K. Massri, et al. [NA62 coll.], presented at ICHEP2018.

[9] B. Beckford, K. Shiomi, et al. [KOTO coll.], presented at ICHEP2018.

[10] A. J. Buras, D. Buttazzo, J. Girrbach-Noe and R. Knegjens, " $K^{+} \rightarrow \pi^{+} v \bar{v}$ and $K_{L} \rightarrow \pi^{0} v \bar{v}$ in the Standard Model: status and perspectives,” JHEP 1511 (2015) 033 [arXiv:1503.02693 [hep-ph]].

[11] H. Park et al. [HyperCP coll.], "Evidence for the decay $\Sigma^{+} \rightarrow p \mu^{+} \mu^{-}$," Phys. Rev. Lett. 94 (2005) 021801 [hep-ex/0501014].

[12] R. Aaij et al. [LHCb coll.], "Evidence for the rare decay $\Sigma^{+} \rightarrow p \mu^{+} \mu^{-}$," Phys. Rev. Lett. 120 (2018) no.22, 221803 [arXiv:1712.08606 [hep-ex]].

[13] R. Aaij et al. [LHCb coll.], "Observation of $D^{0}$ meson decays to $\pi^{+} \pi^{-} \mu^{+} \mu^{-}$and $K^{+} K^{-} \mu^{+} \mu^{-}$final states,” Phys. Rev. Lett. 119 (2017) no.18, 181805 [arXiv:1707.08377 [hep-ex]].

[14] R. Aaij et al. [LHCb coll.], "Measurement of Angular and CP Asymmetries in $D^{0} \rightarrow \pi^{+} \pi^{-} \mu^{+} \mu^{-}$and $D^{0} \rightarrow K^{+} K^{-} \mu^{+} \mu^{-}$decays," Phys. Rev. Lett. 121 (2018) no.9, 091801 [arXiv:1806.10793 [hep-ex]].

[15] R. Aaij et al. [LHCb coll.], "Search for the rare decay $\Lambda_{c}^{+} \rightarrow p \mu^{+} \mu^{-}$," Phys. Rev. D 97 (2018) no.9, 091101 [arXiv:1712.07938 [hep-ex]].

[16] M. Ablikim et al. [BESIII coll.], "Search for the rare decays $D \rightarrow h\left(h^{\prime}\right) e^{+} e^{-}$," Phys. Rev. D 97 (2018) no.7, 072015 [arXiv:1802.09752 [hep-ex]].

[17] M. Ablikim et al., "Search for the rare decays $J / \psi \rightarrow D^{0} e^{+} e^{-}+$c.c. and $\psi(3686) \rightarrow D^{0} e^{+} e^{-}+$c.c.," Phys. Rev. D 96 (2017) no.11, 111101 [arXiv:1710.02278 [hep-ex]].

[18] M. Ablikim [BESIII coll.], "Search for the rare decay of $\psi(3686) \rightarrow \Lambda_{c}^{+} \bar{p} e^{+} e^{-}+$c.c. at BESIII," Phys. Rev. D 97 (2018) no.9, 091102 [arXiv:1802.04057 [hep-ex]].

[19] V. Khachatryan et al. [CMS and LHCb coll.], "Observation of the rare $B_{s}^{0} \rightarrow \mu^{+} \mu^{-}$decay from the combined analysis of CMS and LHCb data," Nature 522 (2015) 68 [arXiv:1411.4413 [hep-ex]].

[20] K. De Bruyn, R. Fleischer, R. Knegjens, P. Koppenburg, M. Merk, A. Pellegrino and N. Tuning, "Probing New Physics via the $B_{s}^{0} \rightarrow \mu^{+} \mu^{-}$Effective Lifetime," Phys. Rev. Lett. 109 (2012) 041801 [arXiv:1204.1737 [hep-ph]]. 
[21] R. Aaij et al. [LHCb coll.], "Measurement of the $B_{s}^{0} \rightarrow \mu^{+} \mu^{-}$branching fraction and effective lifetime and search for $B^{0} \rightarrow \mu^{+} \mu^{-}$decays," Phys. Rev. Lett. 118 (2017) no.19, 191801 [arXiv:1703.05747 [hep-ex]].

[22] R. Aaij et al. [LHCb coll.], "Measurement of the fragmentation fraction ratio $f_{s} / f_{d}$ and its dependence on B meson kinematics,” JHEP 1304 (2013) 001 [arXiv:1301.5286 [hep-ex]].

[23] R. Aaij et al. [LHCb coll.], "Search for the decays $B_{s}^{0} \rightarrow \tau^{+} \tau^{-}$and $B^{0} \rightarrow \tau^{+} \tau^{-}$," Phys. Rev. Lett. 118 (2017) no.25, 251802 [arXiv:1703.02508 [hep-ex]].

[24] C. Bobeth, M. Gorbahn, T. Hermann, M. Misiak, E. Stamou and M. Steinhauser, " $B_{s, d} \rightarrow l^{+} l^{-}$in the Standard Model with Reduced Theoretical Uncertainty,” Phys. Rev. Lett. 112 (2014) 101801 [arXiv:1311.0903 [hep-ph]].

[25] D. Buttazzo, A. Greljo, G. Isidori and D. Marzocca, "B-physics anomalies: a guide to combined explanations," JHEP 1711 (2017) 044 [arXiv:1706.07808 [hep-ph]].

[26] S. L. Glashow, D. Guadagnoli and K. Lane, “Lepton Flavor Violation in B Decays?,” Phys. Rev. Lett. 114 (2015) 091801 [arXiv:1411.0565 [hep-ph]].

[27] R. Aaij et al. [LHCb coll.], "Search for the lepton-flavour violating decays $\mathrm{B}_{(s)}^{0} \rightarrow e^{ \pm} \mu^{\mp}$," JHEP 1803 (2018) 078 [arXiv:1710.04111 [hep-ex]].

[28] R. Aaij et al. [LHCb coll.], "Evidence for the decay $B_{S}^{0} \rightarrow \bar{K}^{* 0} \mu^{+} \mu^{-}$," JHEP 1807 (2018) 020 [arXiv:1804.07167 [hep-ex]].

[29] J. P. Lees et al. [BaBar coll.], "Measurement of angular asymmetries in the decays $B \rightarrow K^{*} \ell^{+} \ell^{-}$," Phys. Rev. D 93 (2016) no.5, 052015 [arXiv:1508.07960 [hep-ex]].

[30] R. Aaij et al. [LHCb coll.], "Angular analysis of the $B^{0} \rightarrow K^{* 0} \mu^{+} \mu^{-}$decay using $3 \mathrm{fb}^{-1}$ of integrated luminosity,” JHEP 1602 (2016) 104 [arXiv:1512.04442 [hep-ex]].

[31] M. Aaboud et al. [ATLAS coll.], "Angular analysis of $B_{d}^{0} \rightarrow K^{*} \mu^{+} \mu^{-}$decays in $p p$ collisions at $\sqrt{s}=8 \mathrm{TeV}$ with the ATLAS detector," arXiv:1805.04000 [hep-ex].

[32] A. M. Sirunyan et al. [CMS coll.], "Measurement of angular parameters from the decay $\mathrm{B}^{0} \rightarrow \mathrm{K}^{* 0} \mu^{+} \mu^{-}$in proton-proton collisions at $\sqrt{s}=8 \mathrm{TeV}$," Phys. Lett. B 781 (2018) 517 [arXiv:1710.02846 [hep-ex]].

[33] A. M. Sirunyan et al. [CMS coll.], "Angular analysis of the decay $\mathrm{B}^{+} \rightarrow \mathrm{K}^{+} \mu^{+} \mu^{-}$in proton-proton collisions at $\sqrt{s}=8 \mathrm{TeV}$, arXiv:1806.00636 [hep-ex].

[34] S. Descotes-Genon, J. Matias, M. Ramon and J. Virto, "Implications from clean observables for the binned analysis of $B \rightarrow K^{*} \mu^{+} \mu^{-}$at large recoil," JHEP 1301 (2013) 048 [arXiv:1207.2753 [hep-ph]].

[35] R. Aaij et al. [LHCb coll.], "Test of lepton universality using $B^{+} \rightarrow K^{+} \ell^{+} \ell^{-}$decays," Phys. Rev. Lett. 113 (2014) 151601 [arXiv:1406.6482 [hep-ex]].

[36] R. Aaij et al. [LHCb coll.], “Test of lepton universality with $B^{0} \rightarrow K^{* 0} \ell^{+} \ell^{-}$decays," JHEP 1708 (2017) 055 [arXiv:1705.05802 [hep-ex]].

[37] S. Wehle et al. [Belle coll.], "Lepton-Flavor-Dependent Angular Analysis of $B \rightarrow K^{*} \ell^{+} \ell^{-}$," Phys. Rev. Lett. 118 (2017) no.11, 111801 [arXiv:1612.05014 [hep-ex]].

[38] W. Altmannshofer, C. Niehoff, P. Stangl and D. M. Straub, "Status of the $B \rightarrow K^{*} \mu^{+} \mu^{-}$anomaly after Moriond 2017,” Eur. Phys. J. C 77 (2017) no.6, 377 [arXiv:1703.09189 [hep-ph]].

[39] I. Bediaga et al. [LHCb coll.], "Physics case for an LHCb Upgrade II - Opportunities in flavour physics, and beyond, in the HL-LHC era," arXiv:1808.08865. 